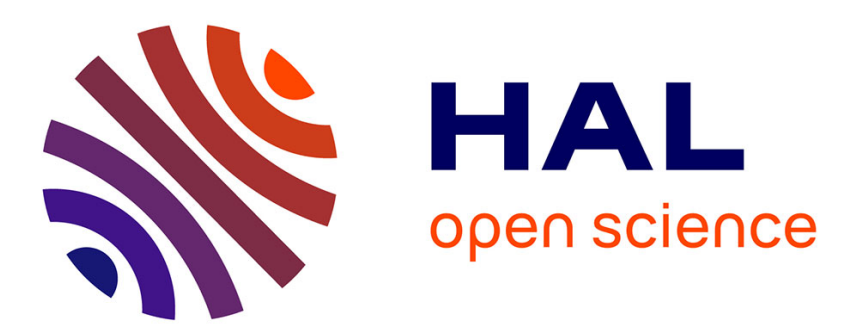

\title{
Study on thermal fragmentation characteristics of a superheated alumina droplet
}

J.A. Zambaux, L. Manickam, R. Meignen, W.M. Ma, S. Bechta, S. Picchi

\section{To cite this version:}

J.A. Zambaux, L. Manickam, R. Meignen, W.M. Ma, S. Bechta, et al.. Study on thermal fragmentation characteristics of a superheated alumina droplet. Annals of Nuclear Energy, 2018, 119, pp.352-361. 10.1016/j.anucene.2018.05.029 . hal-02881810

\section{HAL Id: hal-02881810 https://hal.science/hal-02881810}

Submitted on 16 Jul 2020

HAL is a multi-disciplinary open access archive for the deposit and dissemination of scientific research documents, whether they are published or not. The documents may come from teaching and research institutions in France or abroad, or from public or private research centers.
L'archive ouverte pluridisciplinaire $\mathbf{H A L}$, est destinée au dépôt et à la diffusion de documents scientifiques de niveau recherche, publiés ou non, émanant des établissements d'enseignement et de recherche français ou étrangers, des laboratoires publics ou privés. 


\title{
Study on thermal fragmentation characteristics of a superheated alumina droplet
}

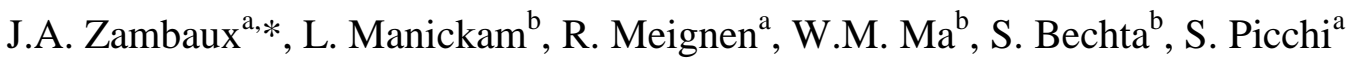 \\ ${ }^{a}$ Institut de Radioprotection et de Sûreté Nucléaire, IRSN/PSN-RES/SAG, BP 3, 13115 Saint-Paul-Lez-Durance Cedex, \\ France \\ ${ }^{b}$ Royal Institute of Technology, Roslagstullsbacken 21, 10691 Stockholm, Sweden
}

\begin{abstract}
In the frame of the European Commission FP7 SAFEST project, IRSN proposed to experimentally investigate the steam explosion triggering mechanisms of a superheated alumina droplet falling into water, through a set of experiments in the Micro Interactions in Steam Explosion Energetics facility (MISTEE) at KTH. Since thermal fragmentation is considered to be a likely process for the triggering of Steam Explosions in the KROTOS tests (performed at CEA) with alumina, the ability of a single droplet of such material to undergo thermally induced fine fragmentation is studied on the MISTEE facility with a close-up visualization.

A series of experiments were conducted, where droplets of molten alumina were discharged into a water pool and potentially exposed to a small pressure wave. The intense interactions were recorded with a high-speed camera along with the pressure in the droplet vicinity.

The ability of alumina to undergo thermal fragmentation is expected to be firstly contingent on the stability of the vapour film enshrouding the melt droplet. The water and melt temperatures may then play a crucial role on the vapour film stability, and therefore on the observation of a steam explosion. Indeed, under high to moderate water sub-cooling conditions, experimental observations indicate that fine fragmentation of the melt can occur when the droplet is exposed to even a weak pressure wave, in the range of $0.15 \mathrm{MPa}$. In contrast, melt fine fragmentation is suppressed at low water sub-cooling conditions (less than $30 \mathrm{~K}$ ), where the formation of a thick vapour film (and large wake) is observed, and which is probably too stable to be destabilized by the weak pressure wave.

The effect of the melt temperature on thermal fragmentation is also assessed. This parameter influences the solidification of the droplet and the strength of the explosion as it determines the available heat energy. In the present conditions, fine fragmentation of melt occurred even at quite low melt superheat $(\approx 60 \mathrm{~K})$. For a high melt superheat (above $200 \mathrm{~K}$ ) a very energetic spontaneous steam explosion was observed. A physical analysis on the debris particles acquired indicates a mass median diameter of $\approx 100 \mu \mathrm{m}$, comparable to the one observed in the KROTOS alumina experiments.

The MISTEE experimental results are finally used to assess the heat and mass transfer modelling of the coolant during the fragmentation process in the FCI code MC3D.
\end{abstract}

Keywords: Steam Explosion, Thermal fragmentation, Alumina, MC3D

\section{Introduction}

A severe nuclear accident can involve high temperature molten materials, including the nuclear fuel, called corium, poured into the coolant fluid. The mixing process that then occurs between the two is generally called FCI (Fuel-Coolant Interaction). FCI may involve two steps. In the first one, the mixing occurs quite quiescently, involving melt fragmentation at the scale of some millimetres. Under some conditions, this pre-mixing phase can be interrupted by a strong explosion, called steam explosion, where the thermal energy is transformed massively into mechanical energy, due to a very fine fragmentation process of the melt. This type of very energetic phenomenon leads to the formation of shock waves and can endanger the structures and the containment of the radioactive materials. The explosion phase happens when the pressure build-up due to both the void production and the coolant properties modifications caused by the temperature increase is faster than the time needed for the system to release the pressure. State-of-the-art reviews on experimental and analytical studies of steam explosion can be found in [1], [2], [3], [4] and [5].

The steam explosion is initiated locally by some triggering events that are still not very well known and understood. Several mechanisms have been identified and studied, among which, the so-called thermal fragmentation. This specific phenomenon leads to the fast fragmentation (within some milliseconds) of a hot liquid droplet into fine fragments while under stable hydrodynamic conditions (i.e. low Weber number). The fragmentation occurs generally in 2 or 3 steps, in an apparent isotropic way. Several hypotheses have again been 
made to explain this specific phenomenon and the analyses have eventually converged towards a mechanism first proposed and modelled by Kim and Corradini [6]. The authors postulated that, under a destabilization of the vapour film through a Rayleigh-Taylor mechanism, small jets of coolant could penetrate the droplet, vaporize and induce the fragmentation. A thorough analysis of thermal fragmentation is given by Lamome and Meignen [7], based on the experimental results and studies made by Nelson and Duda [8][9]. Lamome and Meignen [7] indicated that the phenomenon in fact could be initiated, for a hot droplet initially enshrouded in a vapour film, by local quasi liquid-liquid contacts (the real contact being thermodynamically impossible) between the hot material and the coolant. These contacts would lead to very high local heat and mass transfers and pressurizations, and consequently, through again a Rayleigh-Taylor mechanism, to instabilities of the interface and then fragmentation. The precise development of the fragmentation is nevertheless still partially known as it is clear that analytical modelling reaches rapidly some limits, whereas more direct numerical investigation is still out of reach.

The MISTEE facility at KTH allows studying the thermal fragmentation of a single hot droplet of molten material falling into water in the film boiling regime. A small pressure wave (around 1 or 2 bars) can be applied to the droplet in order to destabilize the vapour film and trigger the thermal fragmentation. Experiments within MISTEE have been made for several kinds of materials with relatively low melting temperature such as tin [10][11] or the binary oxide $\mathrm{WO}_{3}-\mathrm{CaO}$ [12]. Typically, during the thermal fragmentation, between two and three cycles of expansion and collapse of a vapour bubble around the droplet are observed, indicating two to three cycles of fragmentation. These observations are in accordance with Nelson and Duda [8][9] findings with iron oxide. However, X-ray measurements in MISTEE indicated that the droplet fragmentation occurs during the second and third cycles [10].

Within the framework of nuclear power plant safety R\&D, a number of steam explosion experiments have been conducted with various corium simulant and prototypic materials to understand the thermal-hydraulic process that govern the explosivity. In the KROTOS experiments at CEA, spontaneous steam explosions were observed with alumina $\left(\mathrm{Al}_{2} \mathrm{O}_{3}\right)$, while no explosion occurred with prototypic corium in similar conditions [13]. It is now largely admitted that the large loads observed with alumina are mainly due to its low density which generates large droplets and thus low void and slow solidification. Concerning the void, for a sub-cooling of around $80^{\circ} \mathrm{C}$, a void fraction around $2 \%$ with alumina is reported in [13] while it is approximately 10 times bigger with corium. As for the solidification, although no specific measurement of the solidification rate has been made, the solidification effect is admitted to be negligible during the course of the KROTOS experiments, as shown in [14]. Still, there are several other questions related to this material effect that are not answered yet, in particular the triggering mechanism, and that can be addressed with MISTEE. In the frame of the SAFEST European Commission FP7 project [15], the ability of alumina to undergo thermal fragmentation has thus been studied in the MISTEE facility. The melting temperature of alumina is significantly higher $\left(2054^{\circ} \mathrm{C}\right.$ as given in [16]) than the range of temperatures previously used in MISTEE (see [10], [11] and [12]) and the facility was accordingly upgraded. This series of experiments is thus also a first step toward the study of real corium in the MISTEE facility.

The experimental facility and the performed experiments are first presented. The ability of alumina to undergo thermal fragmentation is evidenced. The effect of the water sub-cooling on the explosivity is then studied and the stabilizing role of the vapour film is highlighted. As the melt superheat can also be expected to impact the results, since it modifies the time when the solidification occurs, the effect of this parameter is also analysed. The last section presents briefly a numerical analysis performed with the MC3D code [17]. This study allows a first detailed interpretation of the development of the explosion, as well as a validation, in realistic conditions, of the heat and mass transfer models used in the code.

\section{Experimental measurements}

\subsection{MISTEE facility}

The MISTEE facility is presented on Figure 1. Its main elements are the melting chamber, the confined water pool chamber, the external triggering system and the fast synchronous visual acquisition system. The melting chamber, where approximately $1 \mathrm{~g}$ of solid alumina with $99.8 \%$ purity is initially placed, is a high frequency induction furnace $(20 \mathrm{~kW}, 50$ to $250 \mathrm{kHz})$ and is located at the top of the experimental installation. It is detailed on the right of Figure 1. In order to melt alumina and study the effect of different melt superheat, temperatures typically up to $2300^{\circ} \mathrm{C}$ must be reached. Concentric tubes of advanced ceramics (porous zirconia, alumina and magnesia) surround the tungsten crucible to minimize heat losses. The crucible temperature is monitored by the use of a C-type tungsten rhenium thermocouple mounted from the side of the crucible.

The melt prepared in the tungsten crucible is aerodynamically plugged by a constant purge of inert gas through the $5 \mathrm{~mm}$ crucible nozzle. A fast acting three-way valve is used to control open/close operation for gas flow through the nozzle and also to isolate the furnace from the water pool chamber. When the desired 
temperature is achieved in the crucible, the fast acting three-way valve is released thereby discharging the melt droplet into the water pool chamber. The droplet temperature before entering the water pool is also monitored by a fast two-colour (i.e. emissivity compensated) pyrometer with a spectral range between 1.4 and $1.8 \mu \mathrm{m}$ and uncertainties on the temperature measurements around $12^{\circ} \mathrm{C}$ (Pyrometer on Figure 1).

The water pool chamber is a rectangular plexi-glass tank of dimensions $180 \mathrm{~mm} * 130 \mathrm{~mm} * 350 \mathrm{~mm}$. K-type thermocouples are used to monitor water temperature at two levels (TC on Figure 1).

During the experiments, destabilization of the vapour film and the subsequent (quasi) melt-water contact is initiated by an external triggering system. It is a piston set-up located at the bottom of the water pool chamber. The piston set-up is driven by the rapid discharge of a capacitor bank made of three capacitors of $400 \mathrm{Vdc}$ and $4700 \mathrm{mF}$ each. The triggering system generates a sharp pressure pulse of up to $0.15 \mathrm{MPa}$ with a rising time of $50 \mu \mathrm{s}$. Two pressure transducers have been added to the facility to measure the pressure at the top of the water tank and near the area where the pressure wave hits the droplet (noted PT on Figure 1). One of the transducers being located near the interaction location, it is assumed that the pressure recorded at this point approximates the pressure seen by the droplet. This triggering pressure is known to be a crucial parameter for the occurrence of thermal fragmentation (see [7]).

During the experiments, the fragmentation process is recorded by a fast camera with an acquisition rate up to $0.05 \mathrm{~ms}$ per frame. Details about the acquisition system can be found in [11]. Optical measurements rely largely on the spatial and time resolution, noise and contrast (the grey level difference between two areas, melt and water, that determines if the interfaces can be detected). Typically, thermal fragmentation observed in the tests spans approximately for $2 \mathrm{~ms}$ and a frame rate of at least $6000 \mathrm{fps}$ or above is required to acquire any details. Compensating with low light collectiveness for the low exposure times combined with a low attenuating medium as Alumina significantly thus deteriorate any visual information. Hence, X-ray visualization is not pursued in the current tests with Alumina.

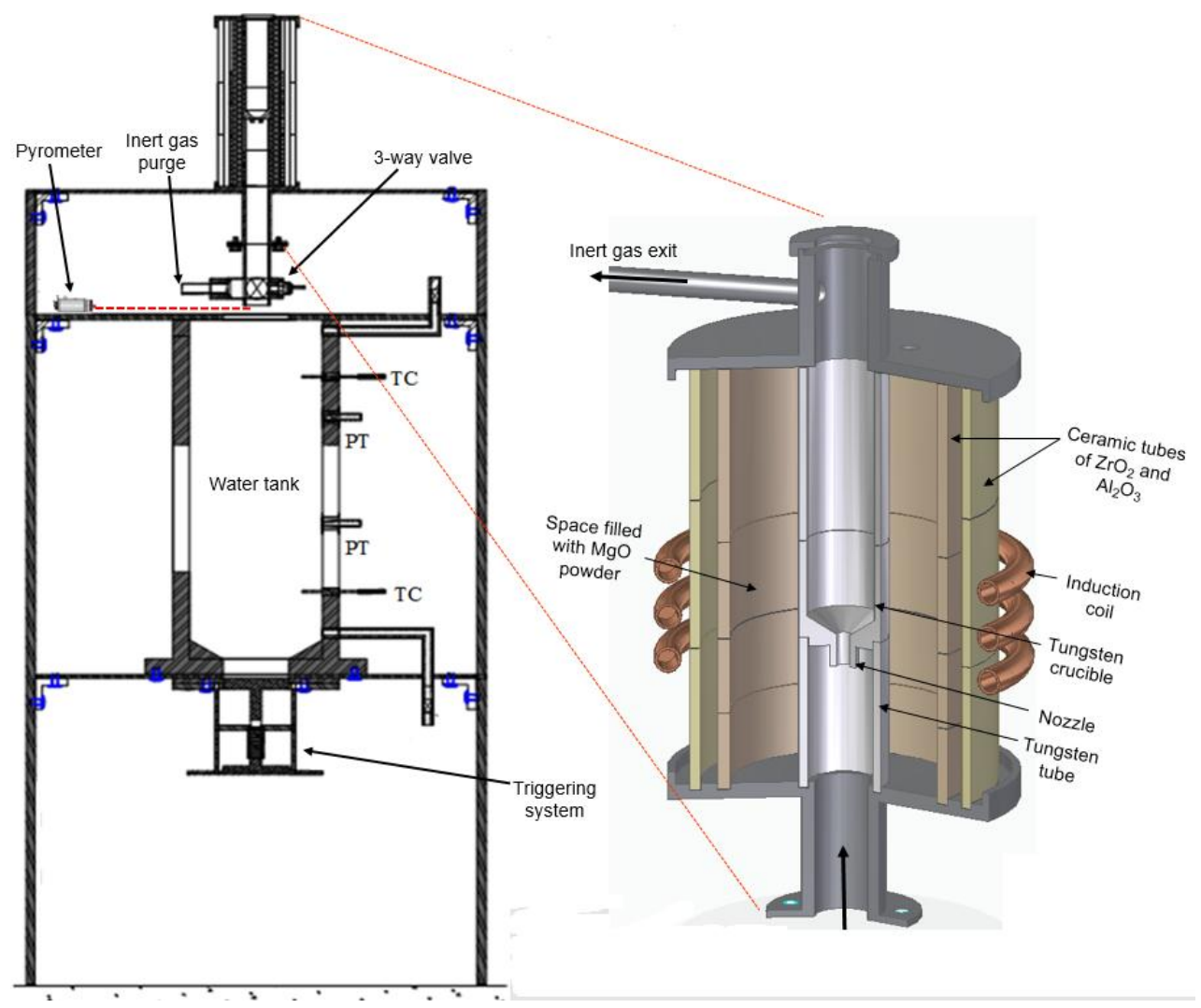

Figure 1 MISTEE facility scheme

\subsection{Post-processing of the experimental results}

The different results analysed here are obtained mainly from the processing of the images given by the camera. The main pieces of information that can be extracted to study the thermal fragmentation mechanisms are presented here.

First, the velocity of the falling droplet is assessed from the estimation of the droplet displacement between two successive images. For most of the experimental cases, with a $1 \mathrm{~g}$ alumina droplet, a velocity around $0.5 \mathrm{~m} / \mathrm{s}$ 
is typically found. Under these conditions, the Weber number is less than 10 and thus, as confirmed by the images, the droplets are hydrodynamically stable.

In order to analyse the fine fragmentation process and the expansion and collapsing history of the vapour film/bubble, it is interesting to plot the equivalent bubble diameter $D$. As a reminder, thermal fragmentation is characterized by a quasi-isotropic behaviour. Therefore the volume of the mixture of melt and coolant can be estimated quite easily during the explosion. From the images given by the high speed camera, the projected area $A_{p}$ occupied by the vapour can be obtained. The camera system was calibrated with a phantom in order to obtain the spatial resolution. Typical spatial resolution for the current tests ranges between 0.1 and $0.2 \mathrm{~mm} / \mathrm{pixel}$ at an uncertainty range of approximately up to 4 pixels. The equivalent diameter corresponding to this area can be calculated by assuming that the shape of the bubble is regular. It is thus given by the equation:

$$
D=\frac{\sqrt{4 A_{p}}}{\pi}
$$

The equivalent diameter is usually normalized by the initial value $D_{0}$, before triggering. The strength of the vapour explosion can be qualified by evaluating a conversion ratio between the available thermal energy and the mechanical energy. The latter is approximated by the work performed by the bubble. During the expansion phase of the bubble, almost all of the energy given by the droplet to the vapour is then used for the work $W(t)$ of the pressure forces $(p . d V)$ exerted on the coolant and can be deduced from the measured equivalent bubble diameter using the Rayleigh-Plesset equation. Thus, the work can be written as:

$$
W(t)=4 \pi \rho_{l} \int_{R_{0}}^{R}\left[R^{3} \ddot{R}+\frac{3}{2} R^{2} \dot{R}^{2}+\frac{2 \sigma R}{\rho_{l}}+4 \frac{\mu_{l} R \dot{R}}{\rho_{l}}\right] d R
$$

Here, $R$ is the bubble equivalent radius $(R=D / 2), \rho_{l}$ is the density of the liquid coolant, $\sigma$ is the surface tension and $\mu_{l}$ is the dynamic viscosity of the coolant. On the other hand, the total thermal energy available from the droplet $\mathrm{E}_{\mathrm{m}}^{0}$ is approximated by the equation:

$$
E_{m}^{0} \approx m_{m}\left(C_{p}\left(T_{m}-T_{l}\right)+H_{\text {fusion }}\right)
$$

$C_{p}$ is the heat capacity of the melt droplet, considered constant for simplicity, $T_{m}$ is its temperature while $T_{l}$ is the water temperature and $H_{\text {fusion }}$ is the fusion enthalpy. Here, $m_{m}$ is the mass of the droplet. The ratio of the thermal energy of the droplet that is used for the explosion (conversion ratio) can therefore be estimated as:

$$
\eta(t) \approx \frac{W(t)}{E_{m}^{0}}
$$

\subsection{Experimental conditions}

For each experiment, the parameters that must be recorded are the water temperature, the melt charge and temperature when it falls into the water (given by the pyrometer on Figure 1), the droplet velocity (deduced from the camera images analysis) and the pressure seen by the droplet (given by the higher pressure transducer PT on Figure 1). All the experiments are performed at atmospheric pressure. This is an important precision as the experiments performed by Nelson \& Duda [8][9], as well as the analysis performed by Lamome \& Meignen [7] highlighted a strong impact of the ambient pressure, in particular between pressures of 1 and 2 bar, with an easier triggering at 2 bar. This effect is not investigated in the present work.

The two main parameters that are varied are the water sub-cooling and the melt superheat. Five kinds of initial conditions are thus defined and summarized in Table 1. The number of exploitable experiments is given in the last column for each type of initial conditions.

Table 1 Experimental conditions

\begin{tabular}{|l|l|l|l|l|}
\hline & Case type & $\begin{array}{l}\text { Water temperature } \\
\text { range }\end{array}$ & $\begin{array}{l}\text { Melt temperature } \\
\text { range }\end{array}$ & $\begin{array}{l}\text { Number } \\
\text { of tests }\end{array}$ \\
\hline W1 & High sub-cooling & $16-21^{\circ} \mathrm{C}$ & $2132-2217^{\circ} \mathrm{C}$ & 10 \\
\hline W2 & Moderate sub-cooling & $45^{\circ} \mathrm{C}$ & $2211^{\circ} \mathrm{C}$ & 1 \\
\hline W3 & Low sub-cooling & $77-87^{\circ} \mathrm{C}$ & $2071-2265^{\circ} \mathrm{C}$ & 3 \\
\hline W4 & Low superheat & $14^{\circ} \mathrm{C}$ & $2117^{\circ} \mathrm{C}$ & 1 \\
\hline W5 & High superheat & $19^{\circ} \mathrm{C}$ & $2259^{\circ} \mathrm{C}$ & 1 \\
\hline
\end{tabular}

Concerning the other parameters, in all the cases, the melt charge is around $1 \mathrm{~g}(+/-0.3$ due to uncertainties during the extraction of the material). The pressure wave amplitude is subjected to variation because of the plug system used in MISTEE and is between 0.06 and 0.2 MPa. Some of the experiments were not triggered among which one spontaneous explosion was observed. This case will be addressed separately in the paper. 


\section{Results}

\subsection{Experimental data analysis for the triggered tests}

The camera recording allows following the explosion process. An example of the droplet and surrounding vapour evolution during the experiment is given on Figure 2. A first visual analysis shows that the droplet behaviour is similar to what was observed for other materials previously studied in MISTEE (see [10],[11] and [12]) and consistent with the observations of Nelson and Duda [8][9]. After meeting the pressure wave, the droplet and surrounding vapour exhibit three successive cycles of isotropic expansion and collapse. In what follows, time $\mathrm{t}=0 \mathrm{~ms}$ corresponds to the beginning of the second cycle which is the most important and expected to be the beginning of the fine fragmentation process.

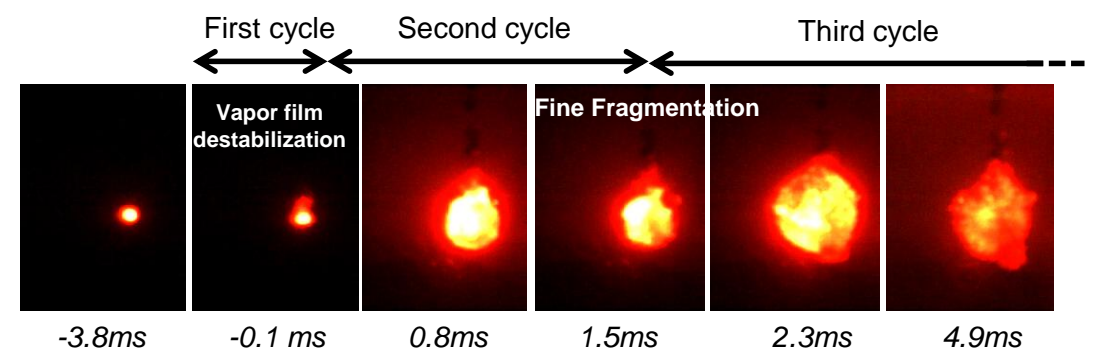

Figure 2 Typical images given by the camera of the droplet dynamic when a steam explosion occurs (with $\mathrm{T}_{1}=18^{\circ} \mathrm{C}$, $\mathrm{T}_{\mathrm{m}}=2217^{\circ} \mathrm{C}, \mathrm{m}_{\mathrm{m}}=0.8 \mathrm{~g}, \mathrm{P}_{\text {trigger }}=0.09 \mathrm{MPa}$ and $\mathrm{U}_{\mathrm{m}}=0.5 \mathrm{~m} / \mathrm{s}$ )

The occurrence of thermal fragmentation was shown by Nelson and Duda [8][9] to depend on the triggering pressure. The impact of the water sub-cooling and the melt superheat on the explosivity, as a function of triggering pressure, is thus plotted on Figure 3(a) and Figure 3(b). The red squares represent the cases where an explosion occurred while the circles are the non-exploding cases.

The observations are consistent with the results obtained by Nelson and Duda [8][9], for similar conditions (i.e. ambient pressure and ambient temperature), with a transition trigger pressure for explosivity that seems to lie between 0.5 and $1 \mathrm{MPa}$ for the highest sub-cooling values (see Figure 3(a)). As for Nelson and Duda, the transition is not very clear and there seems to be some stochastic effect. Figure 3(a) indicates that the water subcooling has a strong impact on the results as no explosion are observed at low sub-cooling (high water temperature) while nearly all the highly sub-cooled cases explode. Although higher trigger pressure might (should) initiate explosion, these results clearly indicate a lower propensity to thermally fragment at low subcooling, consistently with the KROTOS results.

As for the melt sub-cooling, no specific trend can be identified on Figure 3(b). The superheat was always sufficient to initiate explosion when a sufficient sub-cooling was met.

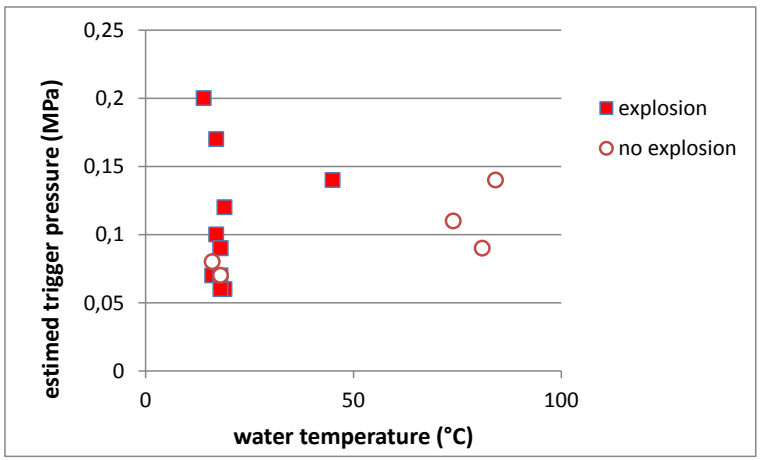

(a) Water temperature and triggering pressure effect

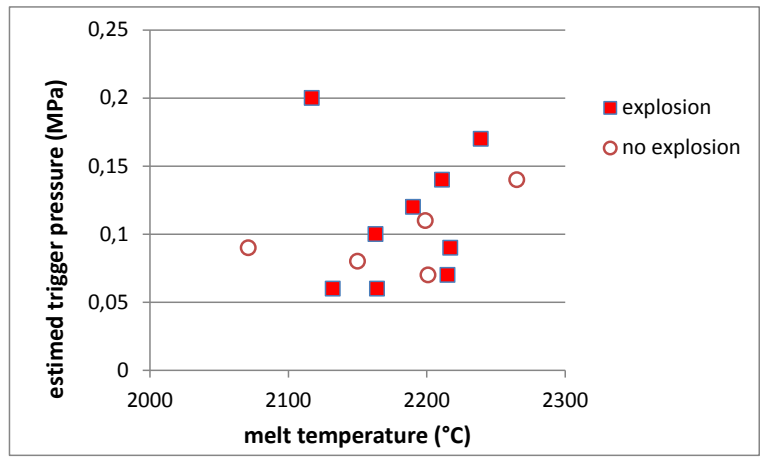

(b) Melt temperature and triggering pressure effect

Figure 3 Explosivity of the experimental cases depending on the temperature and triggering pressure conditions

For all the exploding cases, the conversion ratio can be evaluated from equation (4), by considering the maximum equivalent diameter during the second expansion/collapsing cycle. This point is chosen since the third cycle is not always observed for the different test cases. The calculated conversion ratio for all the exploding experiments ranges from $0.1 \%$ to $0.7 \%$.

Figure 4 shows the conversion ratio as a function of the melt superheat (a) and the triggering pressure (b). The points all correspond to similar high sub-cooling conditions except for the empty square symbol that 
represents moderate sub-cooling condition (see case W2). On Figure 4(a), no clear effect of the melt temperature on the conversion ratio is detected. The available point with moderate sub-cooling does not exhibit a specific behaviour either. As for the effect of the triggering pressure, i.e. the intensity of the destabilizing mechanism, Figure 4(b) shows that the conversion ratio seems to globally increase when the triggering pressure increases. Up to our knowledge, this is the first time that this effect appears and this needs further investigations with a wider trigger pressure range. These results should help in the fine comprehension of the phenomenon.

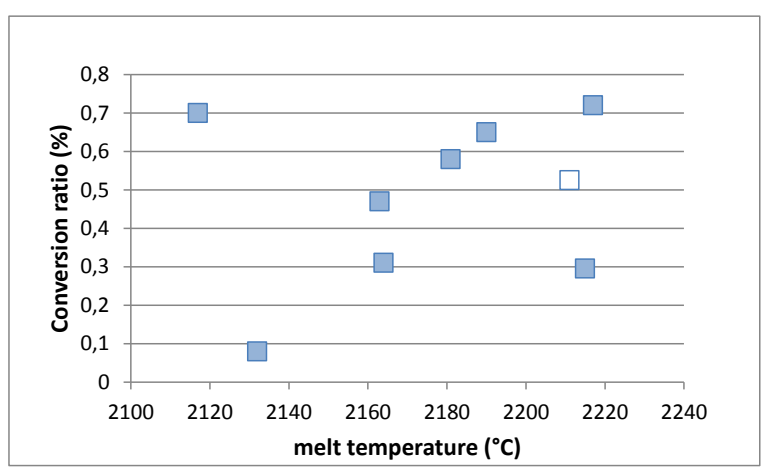

(a) Melt temperature effect

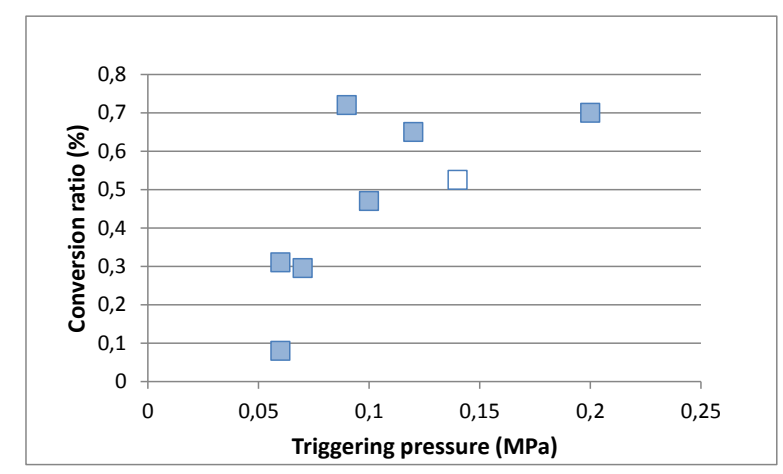

(b) Triggering pressure effect

Figure 4 Plot of the conversion ratio against two initial parameters

At the end of the experiments, the solidified alumina fragments are collected for further analysis. In order to estimate the fragments size, they are subjected to a succession of conventional sieve screens with different pore diameters (up to $1400 \mu \mathrm{m}$ ). The fragments size distribution follows a normal law. The mass median fragment size can thus be evaluated and is referred as D50. On Figure 5, the conversion ratio is plotted against the mass median fragment size. It can be seen that the median size is globally smaller for the highest conversion ratio, as the fragmentation is more energetic. This is expected, as the heat transfer is faster with smaller fragments, as the exchange surface is increased.

The conversion ratios and fragment sizes observed in the MISTEE experiments with alumina are in the range of those observed for the global explosions in the KROTOS tests with alumina (the conversion ratio, estimated according to a different definition, goes up to $2.5 \%$, and the typical fragment size is $100 \mu \mathrm{m}$ [13]). This means that thermal fragmentation is a powerful phenomenon for energy transfer and is likely to be a possible triggering source of the global steam explosions observed in KROTOS tests with Alumina.

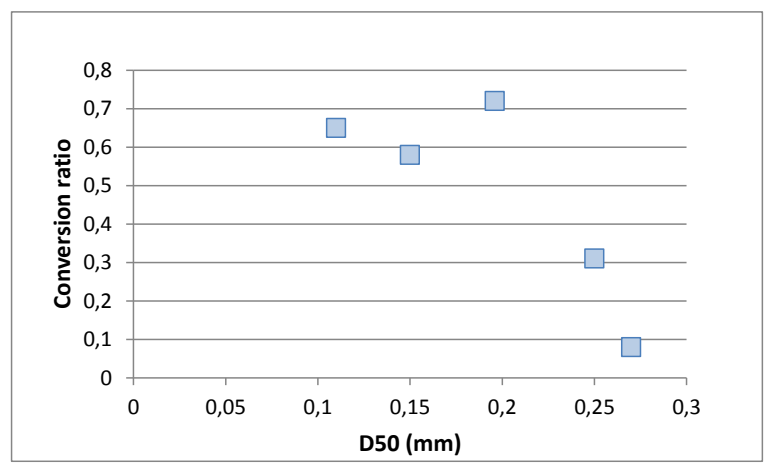

Figure 5 Plot of mass median fragment size against the conversion ratio

It is also worth noting that, compared with the other materials previously studied in MISTEE, alumina gives the smallest fragment size. Hansson et al. [12] showed for example that for eutectic $\mathrm{WO}_{3}-\mathrm{CaO}, \mathrm{D} 50$ is around $0.25 \mathrm{~mm}$ while it is around $0.4 \mathrm{~mm}$ for the non-eutectic binary oxide. This smaller size observed for the fragments with alumina is at the same time the result and the cause of a more energetic explosion. It is likely to be due to the higher temperature, but the different material properties also play a role. To verify this, the thermal energy of alumina can be theoretically compared with the one observed for eutectic $\mathrm{WO}_{3}-\mathrm{CaO}$ for the same material mass, water sub-cooling and melt temperature (using the binary oxide properties given in [12]). For $1 \mathrm{~g}$ of material, a sub-cooling of $80^{\circ} \mathrm{C}$ and a melt temperature of $2150^{\circ} \mathrm{C}$, the thermal energy for alumina is more than twice the one calculated for eutectic $\mathrm{WO}_{3}-\mathrm{CaO}$. 


\subsection{Water sub-cooling effect}

As shown on Figure 3, the water sub-cooling does play an important part on the occurring of thermal fragmentation. This observation is in accordance with the Nelson and Duda [8][9] experiments but also with the global scale KROTOS experiments with alumina where steam explosions were harder to trigger for low subcooling conditions [13] (but yielded the strongest explosions).

When the water temperature is close to the saturation conditions, the vapour production is expected to be higher. The MISTEE images show that, under those conditions, a thick vapour film and wake appear at the rear of the droplet. Figure 6 shows images for one case with a low sub-cooling. The volume of void estimated from the camera images is approximately 6 times the volume of the droplet. The vapour film and wake can clearly be identified from the images. One bubble is periodically detached from the wake and condensed in the liquid. Such a visualisation cannot be done for higher water sub-cooling as the film is too thin and hidden by the light emitted by the hot droplet.
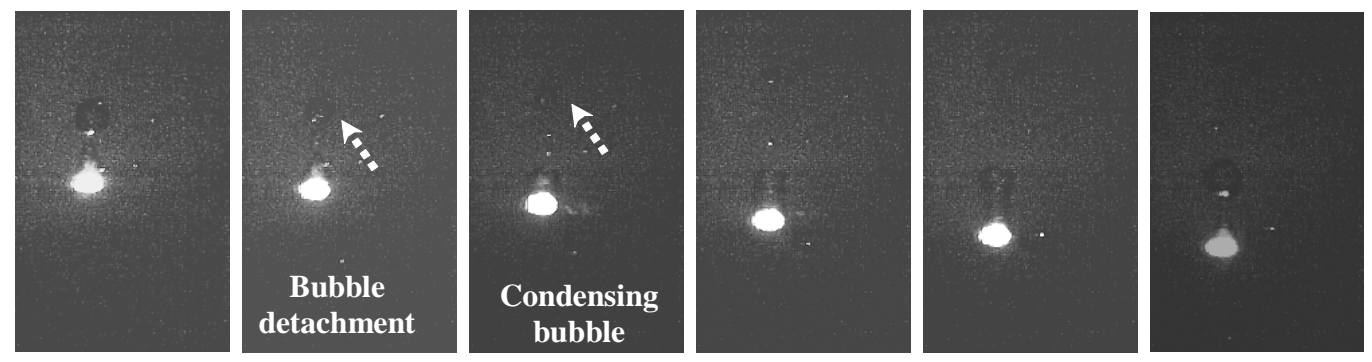

Figure 6 Time evolution of the alumina droplet and surrounding vapour for low sub-cooling conditions (with $\mathrm{T}_{1}=86^{\circ} \mathrm{C}$,

$$
\mathrm{T}_{\mathrm{m}}=2170^{\circ} \mathrm{C}, \mathrm{m}_{\mathrm{m}}=0.9 \mathrm{~g} \text { and } \mathrm{U}_{\mathrm{m}}=0.4 \mathrm{~m} / \mathrm{s} \text { ) }
$$

When the sub-cooling decreases, the vapour film becomes thicker and more stable. The pressure wave needed to trigger the thermal fragmentation process has thus to be increased in order to disturb the vapour film. In the frame of the MISTEE experiments, the triggering pressure is below $0.15 \mathrm{MPa}$ for the low sub-cooling cases (see Figure 3(a)), which seems too weak to destabilize the thick vapour film.

\subsection{Self-triggering event analysis}

A spontaneous steam explosion was observed for a melt superheat of $215^{\circ} \mathrm{C}$ and a water temperature of $19^{\circ} \mathrm{C}$. Figure 7 shows the visualisation of the explosion given by the camera. The steam explosion seems very energetic compared to what was observed for the triggered explosion as the exploding droplet is bigger than the frame of the camera. The MISTEE test-section was destroyed during this experiment.

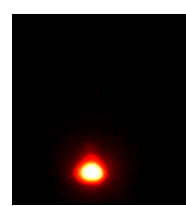

$-14.5 m s$

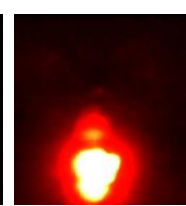

$-1.45 \mathrm{~ms}$

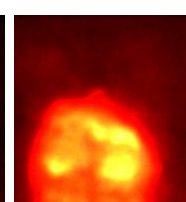

Oms

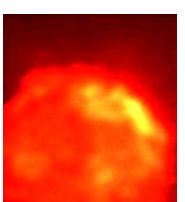

$3.4 m s$

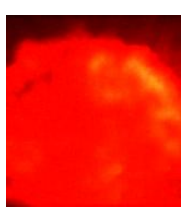

$5.75 \mathrm{~ms}$

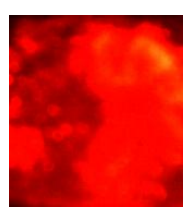

$7.8 m s$

Figure 7 Spontaneous Steam explosion observed for the self-triggering case

Since the fragmentation process is not entirely captured by the camera, it is not possible to evaluate properly the equivalent bubble diameter after a given time and the conversion ratio. The equivalent diameter can be assessed approximately up to the middle of the second cycle. Compared with other triggered cases with similar sub-cooling and melt charge conditions, the equivalent bubble diameter is increased by nearly $42 \%$ when the explosion occurs (around $7 \mathrm{~mm}$ instead of $4 \mathrm{~mm}$ ).

This spontaneously exploding case shows that alumina can easily exhibit spontaneous steam explosion under certain conditions, and strengthen the hypothesis of thermal explosion as an initiating event.

\section{Numerical analysis}

The MISTEE experiments provide data that can be used for the validation and calibration of numerical models in various ways. In particular, it can help to assess MC3D, the IRSN multiphase thermal-hydraulic software for nuclear safety studies, mostly used for the evaluation of FCI. Here, the first results of a numerical 
on going study, comparing the MISTEE experiments with alumina and the MC3D results are reported. The objective here is twofold:

- giving an interpretation of the experiments

- assessing the heat and mass transfers modelling in the code

It must be highlighted that, from various available experiments, the heat transfer from the fragment to the surroundings is rather well known, with an empirical factor of uncertainty around 2 . What is still very uncertain is what is happening to this heat transfer, in particular the amount which is directly passed to water and the one participating to direct vaporization [3][5]. In the MC3D model [5], a large amount of the heat is used to produce vapour which, once transformed into bubbles, can in turn condense. It is a particularly important challenge for steam explosion modelling to be able to assess this model and, possibly, adjust it. The MISTEE experiments give a unique opportunity to do so in unambiguous and realistic conditions (however, only at low pressure conditions).

\subsection{Thermal fragmentation model}

The thermal fragmentation model available in MC3D is parametric. Our objective is not to assess the fragmentation model itself, but the associated heat and mass transfers. To do so, the fragmentation characteristics (fragmentation rate, fragment diameter) are prescribed and adjusted to the experimental ones. After the fragmentation is triggered at $t=t_{f r, 0}^{t h}$, the fragmentation rate $\Gamma_{f r}^{t h}$ follows a cos law:

$$
\Gamma_{f r}^{t h}=C_{f r}^{t h} \frac{1}{\Delta t_{f r}} M_{d r} \alpha_{d r} \rho_{d r}\left(1-\cos \left(2 \pi \frac{t-t_{f r, 0}^{t h}}{\Delta t_{f r}}\right)\right)
$$

The fragmentation time $\Delta t_{f r}$ is user-defined as well as the percentage of droplets that participate in the fragmentation $M_{d r}$. The multiplicative coefficient $C_{f r}^{t h}$ can also be modified to adjust the fragmentation rate. In the model, the melt droplet is fragmented into spherical fragments with identical user-defined diameter. For the present study, the thermal fragmentation starts spontaneously at $\mathrm{t}=0$.

\subsection{Geometry and initial conditions}

In the preliminary work, in order to ensure small calculation time, the tank geometry is simulated by a cylinder with a total volume equal to the experimental rectangular tank. Therefore, a $2 \mathrm{D}$-axisymmetric domain, meshed regularly, can be considered. The height of the domain is $350 \mathrm{~mm}$ with an $86 \mathrm{~mm}$ radius while the cells size is $6 \mathrm{~mm}$.

Initially, the domain is nearly filled with water. The pressure is fixed at the atmospheric pressure on the upper bound of the domain. In one cell, on the geometry vertical axis a given amount of alumina droplets is placed. Figure 8 presents the domain and the initial conditions. Here the blue area is the water; the white part is gas while the red one marks the presence of alumina droplets. Obviously, these initial conditions are quite crude but they are sufficient for the study current objectives.

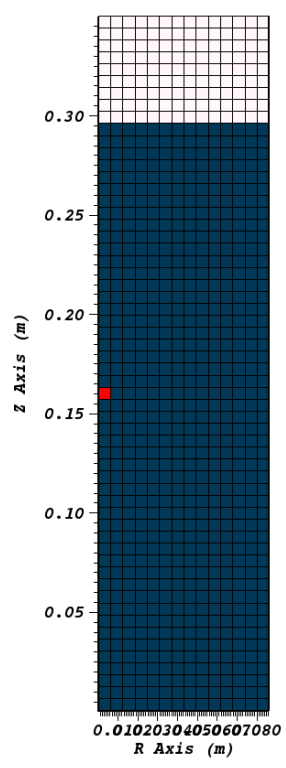

Figure 8 : Numerical domain considered 
The initial conditions used for the numerical analysis are taken from one among the highly sub-cooled experimental cases (82.1 K of sub-cooling) and are summed up on

Table 2. The initial volume fraction of droplets is calculated in order to have the same mass of alumina as in the experiment.

Table 2 : Initial conditions (from test W1)

\begin{tabular}{|l|l|l|l|l|l|l|}
\hline Parameters & $\begin{array}{l}\text { Water } \\
\text { temperature } \\
(\mathrm{K})\end{array}$ & $\begin{array}{l}\text { Droplets } \\
\text { Temperature } \\
(\mathrm{K})\end{array}$ & $\begin{array}{l}\text { Droplets } \\
\text { velocity } \\
\left({\mathrm{m} . \mathrm{s}^{-1}}^{-1}\right.\end{array}$ & $\begin{array}{l}\text { Droplets } \\
\text { diameter } \\
(\mathrm{m})\end{array}$ & $\begin{array}{l}\text { Melt mass } \\
(\mathrm{kg})\end{array}$ & $\begin{array}{l}\text { Droplets } \\
\text { volume } \\
\text { fraction }\end{array}$ \\
\hline Initial values & 291 & 2490 & 0.5 & $8.10^{-3}$ & $8.10^{-4}$ & 0.32 \\
\hline
\end{tabular}

In the MISTEE experiment, the alumina droplet is completely fragmented. Therefore, in the MC3D model, the parameter $M_{d r}$ is chosen equal to 1 . The thermal fragmentation time is set to $1 \mathrm{~ms}$. To ensure that all the droplet is fragmented at the end of the calculation, the fragmentation law has to be adjusted and the multiplicative coefficient $C_{f r}^{t h}$ is set to 10 . The fragments size is fixed at $200 \mu \mathrm{m}$, i.e. the median mass diameter found experimentally for the considered case. There is an uncertainty on the representative size of the distribution in FCI conditions. It can be noted that MC3D has recently been upgraded to be able to represent a drop size distribution through a MUSIG like model [18]. However, in the present case, this model could not be used without some code changes not needed for this qualitative preliminary analysis.

\subsection{Results of the analysis}

Once the fragmentation is started in the model, the fragments are produced without any specific velocity. As soon as they are in contact with water, they produce vapour, which finally forms a big bubble similar to the experiment. The displacement of the fragment is solely due to the hydrodynamic interactions with the ambient fluid. In reality, the fragments should have an initial small outward velocity, due to the fragmentation process itself. It must also be noticed that the latest fragments may be formed within a large void. Consequently, they should weakly interact.

The total volume of void produced around the droplet and fragments is estimated and transformed into an equivalent bubble diameter that can be compared to the experimental result. Figure 9 shows the obtained numerical and experimental results over time for the same initial conditions with the default parameters and models of the MC3D-EXPLO code (version 3.8). The experimental results are plotted from the second expansion/collapsing cycle since it is the supposed to be the beginning of the fragmentation process.

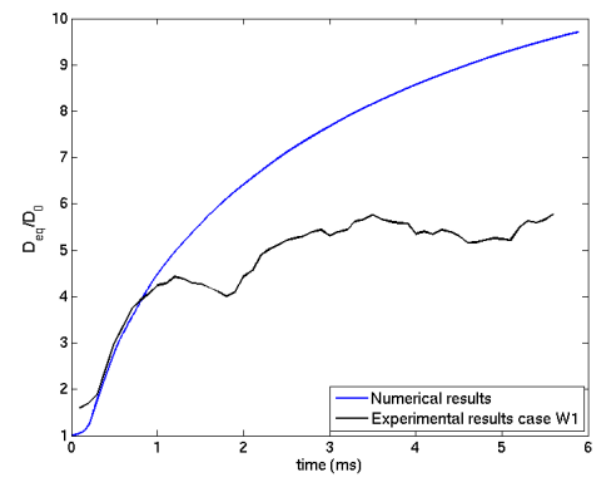

Figure 9 : Equivalent bubble diameter found experimentally and numerically

The results show that, during the first simulated millisecond, when the fragmentation occurs, the bubble grows similarly to what is observed experimentally. Then, instead of stabilizing, it keeps slowly increasing over the simulation time. Hence these observations are in qualitative agreement but tend to show that the heat transfer between the melt and the water is overestimated while the condensation of the vapour may be underestimated.

The heat transfer between the molten material and the water is calculated from the Epstein and Hauser correlation [19] for film boiling heat transfer around a sphere. The film boiling heat transfer is reminded here:

$N u_{F B}=\frac{2}{\beta} \sqrt{\frac{3}{2} R e_{l}}\left[\frac{1}{24 A}+\left(\frac{2}{\pi}\right)^{2}\left(\frac{B}{A}\right)^{4}\right]^{\frac{1}{4}}$ with $\beta=\left[\left(\frac{\rho_{v}}{\rho_{l}}\right)^{\frac{1}{2}} \frac{v_{v}}{v_{l}}\right]^{\frac{1}{2}}, A=\frac{C_{p_{v}}\left(T_{m}-T_{s a t}\right)}{P r_{v} L}$ and $B=\beta \frac{\lambda_{l}}{\lambda_{v}} \frac{C_{p_{v}}\left(T_{s a t}-T_{l}\right)}{P r_{v} L} \sqrt{P r_{l}}$ 
The 1 and $v$ subscript stands for the liquid and vapour phases, $R e_{l}$ is the Reynolds number, $\rho$ the density, $\lambda$ the heat conductivity, $v$ the cinematic viscosity, $C_{p}$ is the heat capacity, $P r$ is the Prandtl number and $L$ is the latent heat of evaporation.

Previous analyses on other experimental results [20] showed that this correlation can overestimate the heat transfer by nearly two times when the sub-cooling is important (more than $80 \mathrm{~K}$ in the experiments presented in [20]). Thus, the first conclusions drawn from the MISTEE numerical results seems to be in accordance with these previous observations on the heat transfer between fine fragments and water in the film boiling regime.

There are also uncertainties concerning the evaluation of the heat transfer between the vapour and the liquid water. In MC3D, the vapour is assumed to form bubbles and the heat transfer is calculated from classical correlations. In the configuration studied here, there is one big expanding bubble and the usual correlations may not be applicable. Concerning future studies, the MISTEE results provide the opportunity to correct these transfers.

A new calculation is made with the transfer by film boiling around fragments multiplied by a 0.5 coefficient. The new results obtained are presented on Figure 10. The equivalent diameter evolution is closer to the experimental results. Thus, the hypothesis made on the film boiling heat transfer seems reasonable.

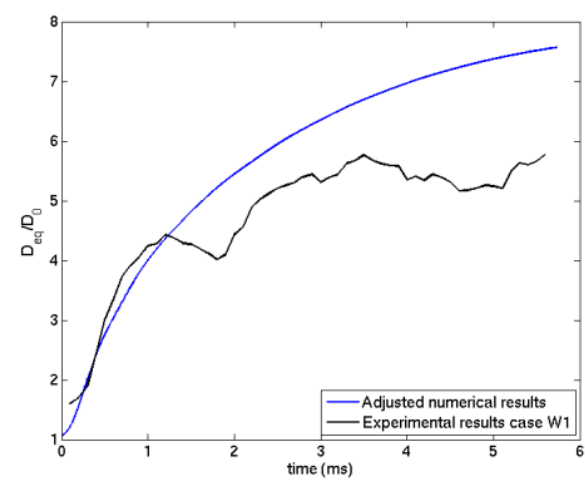

Figure 10 : Equivalent diameter obtained from the experiments and the adjusted simulations

The maximum equivalent diameter increase is notably reduced compared with the previous results, although it is clear that some adjustments are still needed. Some other parameters effects, as the fragments size distribution, have not been considered here and are expected to play also a part.

The numerical results can give access to the evolution of the vapour bubble but also complementary information as the fragments position. Figure 11 presents for different times the fragment volume fraction repartition. The black line corresponds to the liquid/vapour interface.
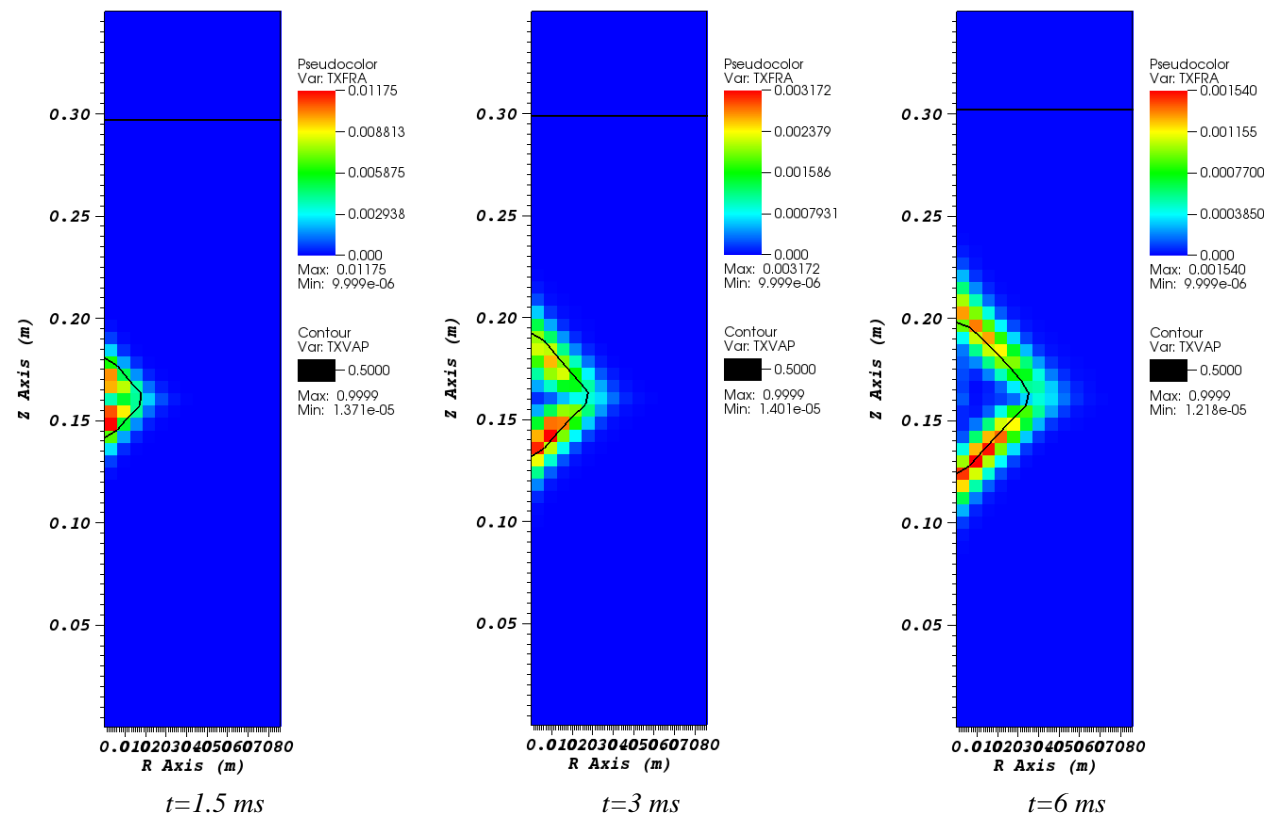

Figure 11 : Alumina fragments volume fraction and liquid/vapour interface 
Figure 11 shows the vapour bubble when it is expanding due to the heat exchange with the fragments. It must be noted that the fragments temperature remains really high during the simulations time frame (above $2300 \mathrm{~K}$ for fragments volume fraction above 0.001). In the numerical simulations, the fragments are mainly ejected toward the bubble surface and follow it closely, which is what was previously observed with the X-ray measurement for $\mathrm{WO}_{3}-\mathrm{CaO}$ in the previous MISTEE experiments [12].

From the numerical results, the shape of the vapour bubble is not really spherical. Preliminary simulations with a finer mesh show this is mainly due to the shape of the initial droplet distribution (in only one cell).

This preliminary simple numerical analysis with MC3D shows that, with some thermal laws adjustment, the thermal fragmentation model gives results in the same range as the one obtained in the MISTEE experiments with alumina. Although the model needs refinement and a more complete validation, this short analysis shows that the MISTEE experiments with alumina do provide very valuable data for model validation.

\subsection{Complementary calculations}

Three supplementary calculations were performed based on different MISTEE experimental conditions. The model parameters are the same as the one defined in the previous section. Among the three cases selected, one has similar conditions compared with the case simulated in the previous section but a smaller mean fragments diameter (case W1.2 from the experiments). Another case corresponds to low sub-cooling conditions (case W3). No explosion was recorded in the corresponding experiment. The thermal fragmentation was thus not triggered in the numerical simulation performed for this case. The last case corresponds to the self exploding event (case W5) despite lacking experimental data. Since fragments could not be collected due to the damages of the MISTEE installation, an arbitrary size of $75 \mu \mathrm{m}$ has been chosen for the fragments (the typical mean size used by default in MC3D and based on the KROTOS experiments results [13]). For the three supplementary cases, the droplet mass and diameter are the same as for the case previously considered. The other initial conditions are summarised on Table 3 .

Table 3: Initial conditions for the supplementary calculations

\begin{tabular}{|l|l|l|l|l|}
\hline Case & $\begin{array}{l}\text { Water } \\
\text { temperature }(\mathrm{K})\end{array}$ & $\begin{array}{l}\text { Droplets } \\
\text { Temperature }(\mathrm{K})\end{array}$ & $\begin{array}{l}\text { Droplets } \\
\text { velocity }\left(\mathrm{m}_{\mathrm{s}} \mathrm{s}^{-1}\right)\end{array}$ & $\begin{array}{l}\text { Mean fragments } \\
\text { size }(\mu \mathrm{m})\end{array}$ \\
\hline W1.2 & 289 & 2454 & 0.46 & 150 \\
\hline W3 & 360 & 2520 & 0.46 & No explosion \\
\hline W5 & 292 & 2532 & 0.5 & No data \\
\hline
\end{tabular}

The mean bubble diameter is plotted on Figure $\mathbf{1 2}$ for the three supplementary calculations (cases W1.2, W3 and W5) along with the results obtained in the previous section (case W1). For all the cases, the experimental results are presented with a continuous line while the numerical results are given with a dashed line. It is worth noting that for the case W5, only the first millisecond is available from the experiments.

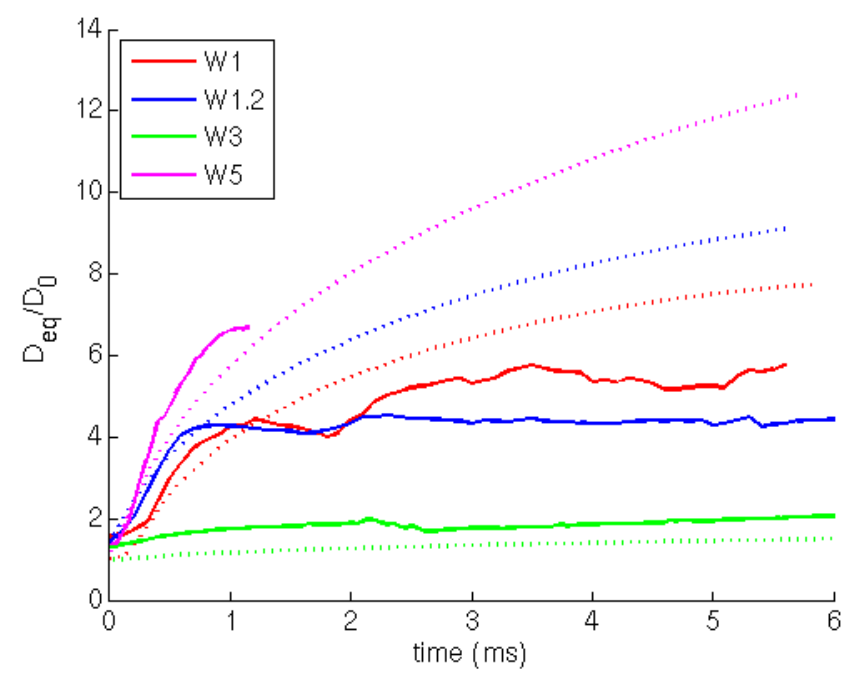

Figure 12 : Evolution of the mean bubble diameter from the numerical and experimental results for four sets of initial conditions 
Accordingly to what was observed in the previous section for only one set of experiments, the proposed adjustment of the thermal laws gives results in the same range as the MISTEE experimental results, either when there is no thermal fragmentation (case W3) or when the thermal fragmentation model is activated (cases W1, W1.2 and W5). Refinements are still needed to better describe the collapsing of the bubble.

These complementary calculations enhance the important role played by the fragment diameters on the numerical results. This parameter is the main difference between case W1 (fragment size of $200 \mu \mathrm{m}$ ) and case W1.2 (fragment size of $150 \mu \mathrm{m}$ ) presented on Figure 12. In MC3D, since this diameter is fixed, its value must be chosen carefully. Moreover, a way to improve the numerical results is to take into account the fact that all the fragment are not at the same size and may not appear at once as there might more than two expansion/collapsing cycles in the experiments.

\section{Conclusions}

To study thermal fragmentation with alumina, the MISTEE facility was successfully adapted for high temperature melting materials. The thermal fragmentation mechanisms observed for alumina are similar to the ones observed for materials with lower melting points such as tin or $\mathrm{WO}_{3}-\mathrm{CaO}$. Between two and three cycles of bubble expansion and collapsing can be seen when the droplet is submitted to a small pressure wave. Due to the higher temperature, bigger equivalent bubble diameter and conversion ratio are observed with alumina compared with the other materials previously considered in MISTEE. For similar melt superheat, with alumina, the maximum bubble diameter is generally between 4 and 5 times the droplet initial diameter while it is below 3 for non-eutectic $\mathrm{WO}_{3}-\mathrm{CaO}$ and around 4 for the eutectic composition. As for the conversion ratio, it is between $0.3 \%$ and $0.7 \%$ with alumina while it is below $0.1 \%$ for non-eutectic $\mathrm{WO}_{3}-\mathrm{CaO}$ and between $0.1 \%$ and $0.5 \%$ for the eutectic material.

The water sub-cooling does play an important part in the occurrence and the strength of the thermal fragmentation as it directly influences the void production which has a stabilizing effect and the melt viscosity. For a high sub-cooling of the water $\left(81^{\circ} \mathrm{C}\right)$ and a high superheat of the alumina melt $\left(205^{\circ} \mathrm{C}\right)$, a spontaneous steam explosion was observed, sensibly stronger than the triggered explosions.

The use of alumina within the MISTEE facility is the first step in studying real corium. Further studies with different materials, such as pure zirconium, have now to be performed. Such analysis can give promising results, notably on the following of the oxidation and on its effect on the thermal fragmentation process.

As shown here, the MISTEE experiments can also be used to complete and validate numerical models on a simple case in the MC3D software. This type of studies is the first step to improve the modelling of steam explosion on bigger scale configurations and also to provide a better understanding of the fragmentation process.

\section{Acknowledgments}

The authors acknowledge the financial support within the European Commission FP7 SAFEST project.

[1] M. L. Corradini, B. J. Kim, M. D. Oh, Vapor explosions in light water reactors: A review of theory and modeling. Progress in Nuclear Energy, 22 (1), 1-117, 1988.

[2] D. F. Fletcher, Steam explosions triggering: A review of theoretical and experimental investigation. Nuclear Engineering and Design 155, 27-36, 1995.

[3] R. Meignen, B. Raverdy, M. Buck, G. Pohlner, P. Kudinov, W. Ma, C. Brayer, P. Piluso, S. Hong, M. Leskovar, M. Ursic, G. Albrecht, I. Lindholm, I. Ivanov, Status of steam explosion understanding and modelling, Annals of Nuclear Energy, 74, 125-133, 2014.

[4] R. Meignen, S. Picchi, J. Lamome, B. Raverdy, S. Castrillon Escobar, G. Nicaise, The challenge of modelling fuel-coolant interaction : Part I - Premixing, Nuclear Engineering and Design, 280, 511-527, 2014.

[5] R. Meignen, B. Raverdy, S. Picchi, J. Lamome, The challenge of modelling fuel-coolant interaction: Part II Steam explosion, Nuclear Engineering and Design, 280, 528-541, 2014.

[6] B. Kim, M.L. Corradini, Modelling of small-scale single droplet fuel/coolant interactions, Nuclear Science and Engineering, 98, 16-28, 1988.

[7] J. Lamome, R. Meignen, On the explosivity of a molten drop submitted to a small pressure perturbation, Nuclear Engineering and Design, 238, 3445-3456, 2008. 
[8] L.S. Nelson, P.M. Duda, Steam explosion experiments with single drops of iron oxide, NUREG CR-2285, September, 1981

[9] L.S. Nelson, P.M. Duda, Steam explosion experiments with single drops of iron oxide: PART II: parametric studies, NUREG CR-2718, April, 1985

[10] H.S. Park, R. C. Hansson, B.R. Sehgal, Fine fragmentation of molten droplet in highly subcooled water due to vapour explosion observed by X-ray radiography, Experimental Thermal and Fluid Science, 29, 351361,2005 .

[11] R. C. Hansson, H.S. Park, T.N. Dinh, Simultaneous high speed digital cinematographic and X-ray radiographic imaging of a intense multi-fluid interaction with rapid phase changes, Experimental thermal fluid and science, 33, 754-763, 2009.

[12] R. C. Hansson, T.N. Dinh, L. T. Manickam, A study of the effect of binary oxide materials in a single droplet vapor explosion, Nuclear Engineering and Design 264, 168-175, 2013.

[13] I. Huhtiniemi, D. Magallon, H. Hohmann, Results of recent KROTOS FCI tests: alumina versus corium melts. Nuclear Engineering and Design, 189, 379-389, 1999.

[14] M. Uršič, M. Leskovar, B. Mavko, Simulations of KROTOS alumina and corium steam explosions experiments: Applicability of the improved solidification influence modelling, Nuclear Engineering and Design, 246, 163-174, 2012.

[15] http://cordis.europa.eu/project/rcn/188519_en.html

[16] S. J. Schneider, Cooperative Determination of the Melting Point of Alumina, Pure and Applied Chemistry, 21, 117-122, 1979.

[17] G. Berthoud, M. Valette, Development of a multidimensional model for the premixing phase of a fuelcoolant interaction, Nuclear Engineering and Design, 149, 409-418, 1994.

[18] E. Krepper, D. Lucas, T. Frank, H-M. Prasser, P. J. Zwart, The inhomogeneous MUSIG model for the simulation of polydispersed flows, Nuclear Engineering and Design, 238, 1690-1702, 2008.

[19] M. Epstein, G. M. Hauser, Subcooled forced-convection film boiling in the forward stagnation region of a sphere or a cylinder, International Journal of Heat and Mass Transfer, 23, 179-189, 1980.

[20] G. Berthoud, L. Gros D'Aillon, Film boiling heat transfer around a very high temperature thin wire immersed into water at pressure from 1 to 210 bar: Experimental results and analysis, International Journal of Thermal Sciences, 48, 1728-1740, 2009. 